\title{
Supporting dentists' health and wellbeing - workforce assets under stress: a qualitative study in England
}

\author{
Jennifer E. Gallagher, ${ }^{* 1}$ Fiorella B. Colonio-Salazar² and Sandra White ${ }^{3}$
}

\section{Key points}

Highlights the wide range of contemporary influences on dentists' health and wellbeing, from macro- through meso- to micro-level.

\author{
Raises concerns that dentists, \\ particularly those with high NHS \\ primary care work commitments, \\ are perceiving lack of control in their \\ professional lives.
}

Discusses the implications of retaining the dental workforce in light of the COVID-19 pandemic and its impact on our profession.

\begin{abstract}
Background Multiple determinants influence dentists' health and wellbeing. In light of recent concerns, the aim of this research was to explore contemporary influences on dentists' health and wellbeing in England, drawing on their lived experience.
\end{abstract}

Methods Semi-structured interviews were conducted with a purposive sample of dentists working in England, taking into account age, gender, career stage, work sector, geographical area, position and route of entry to registration. A topic guide, informed by the literature, was used to guide the discourse. Dentists were approached via gatekeepers, supported by snowball sampling. Interviews were recorded and transcribed for analysis. Framework analysis was used, taking an interpretative phenomenological approach to develop theory.

Results Twenty dentists, from a range of backgrounds, participated in this research. While health and wellbeing was reported to be more positive among dentists in their later career, those in their early career and/or with high NHS work commitments shared concerns about their physical, psychological and emotional health. Influences ranged from macro-level issues relating to professional regulation and health systems, through meso-level, notably their workplace and job specification, to micro-level issues relating to their professional careers, relationships and personal life. Dentists highlighted ethical concerns and feeling driven to deliver, together with being demoralised by a survival culture, all leading to perceived lack of control and reduced professional fulfilment. In contrast, being able to deliver quality care, innovate and effect change, as well as being valued for their delivery, were perceived to positively contribute to general health and wellbeing.

Conclusion Dentists have a vital role in providing care for patients, so there are serious public health implications if urgent action is not taken to improve and sustain their health and wellbeing. This study confirms that dentists are affected by multiple contemporary influences, and although greater support is needed for individuals, organisational, system and policy changes may be required to fully address the challenges they face.

'Dean for International Affairs, Newland-Pedley Professor of Oral Health Strategy/Honorary Consultant in Dental Public Health, King's College London, Faculty of Dentistry, Oral \& Craniofacial Sciences, Centre for Host Microbiome Interactions, Denmark Hill Campus, Bessemer Road, London, SE5 9RS, UK; ' $F$ Former PostDoctoral Research Associate, King's College London, UK ; ${ }^{3}$ Former National Lead for Dental Public Health, Health Intelligence, Health Improvement Directorate, Public Health England, Skipton House, Area B, 2nd floor, 80 London Road, London, SE1 6LH, UK.

*Correspondence to: Jennifer Gallagher Email address: jenny.gallagher@kcl.ac.uk

Refereed Paper.

Accepted 7 October 2020

https://doi.org/10.1038/s41415-021-3130-9

\section{Background}

Dentists' health and wellbeing

Our recently published rapid review, ${ }^{1}$ highlighted by the Editor-in-Chief, ${ }^{2}$ reported multiple determinants on dentists' health and wellbeing within the United Kingdom (UK). These ranged from personal and professional issues, moving through job and workplace characteristics to the National Health Service (NHS) dental system and its regulation. While there is powerful recent evidence on health and wellbeing of dentists, ${ }^{3,4,5,6,7,8,9,10}$ much of this research had been conducted a decade or more ago or was quantitative in nature. Increasing quantitative evidence suggests that dentists working at the 'coalface' to deliver NHS contracts are particularly unhappy and many have considered leaving dentistry. ${ }^{11}$ Morale is reportedly poor and reducing, ${ }^{7,8}$ with evidence of NHS dentists reporting their intention to leave or scale down their commitment in the next five years. ${ }^{12}$ Three-quarters of practice owners in England ${ }^{13}$ and across the UK, ${ }^{14}$ are struggling to fill vacancies, raising questions about the sustainability of services.

Professional life generally is presenting challenges to younger generations. Many junior doctors are considering leaving our sister profession, medicine ${ }^{15}$ with health and wellbeing the major reason for doctors to taking break from training. ${ }^{16}$ Given the length 
and cost of dental training, ${ }^{17}$ the prevalence of oral disease with long-term sequelae ${ }^{18,19,20}$ and the importance of supporting professionals in distress, ${ }^{4}$ it is crucial to better understand contemporary influences on dentists' health and wellbeing, to ensure a sustainable healthcare system that is able to meet the needs and demands of patients.

\section{England: dental care system}

Most dentistry is delivered in primary dental care settings by dental teams, in which dentists form the lynchpin. Dental care is increasingly provided by corporate bodies and larger organisations, rather than the traditional model whereby a practice is owned and managed by a principal dentist. England has a distinctive NHS dental care system, which in 2006 moved from a fee-per-item NHS payment system to 'units of dental activity' (UDA), delivered under 'contracts' between providers (one or more dental practices) and NHS England which, despite recommendations for change, ${ }^{21}$ remains in place for the majority. There is significant

\section{Table 1 Characteristics of participants}

\section{Characteristic}

\begin{tabular}{l|l}
\hline Sex & $\begin{array}{l}8 \text { male } \\
12 \text { female }\end{array}$ \\
\hline & UK-qualified 17
\end{tabular}

Route to enter the UK GDC registration list

ORE: 1

EEA-qualified: 1

Overseas-qualified: 1

\begin{tabular}{|c|c|}
\hline Professional type & $\begin{array}{l}\text { Public health: } 1 \\
\text { Primary care setting: } 12 \\
\text { NHS GDS (+ academic): } 5 \text { (Associates) } \\
\text { GDS private (+ academic): } 1 \text { (Specialist) } \\
\text { GDS mixed: } 2 \text { (Principal) } \\
\text { Community Dental Services: } 4 \text { (including two also working in mixed practice } \\
\text { and dental emergency services). } \\
\text { Secondary care: } 3 \\
\text { Hospital + academia (including management or private practice): } 3 \\
\text { (Specialists) } \\
\text { Training posts: } 4 \\
\text { DFT: } 3 \\
\text { SpR: } 1\end{array}$ \\
\hline Geographical area & $\begin{array}{l}\text { London: } 9 \\
\text { Northampton: } 3 \\
\text { Manchester: } 1 \\
\text { Chichester: } 2 \\
\text { Cheddar: } 1 \\
\text { Bristol: } 1 \\
\text { Brighton: } 1 \\
\text { Devon: } 1 \\
\text { Yorkshire: } 1\end{array}$ \\
\hline $\begin{array}{l}\text { Length of time working } \\
\text { since qualification }\end{array}$ & $\begin{array}{l}1 \text { to } 15 \text { years: } 12 \\
16 \text { to } 25 \text { years: } 3 \\
26 \text { and above: } 5\end{array}$ \\
\hline
\end{tabular}

Dental Council (GDC) registration list (ORE UK exam, overseas-qualified, UK-qualified and EEA-qualified), position in the practice (for example, principal, performer/provider), work sector (NHS, private, mixed or other), geographical area (for example, city/town, suburban, rural, mixed, other) and length of time working since qualification $(1-15,16-25$, $26+$ years). Initial subjects for interview were identified through research gatekeepers, ensuring geographical and organisational coverage. This was supported by snowball sampling. ${ }^{24,25}$ The interview topic guide (see online supplementary information) was informed by past research ${ }^{1}$ and professional literature. .,10,26,27 $^{2}$

Interviews were conducted by one female postdoctoral researcher (FCS) trained in qualitative research. While being a qualified dentist, the interviewer, employed as an academic researcher and tutor, was not part of the healthcare system. Interviews were held at a mutually convenient time in the participants' dental practice, or conducted by phone, with the emphasis on building rapport with the respondent and enabling free-flowing discussions.

Individual interviews allowed deep exploration of issues with a high degree of confidentiality, which was important given the possibility to explore personal influences on health and wellbeing. Interviews, lasting 30-75 minutes, were audio-recorded and transcribed verbatim using a confidential transcription service.

Framework methodology was used to facilitate rigorous and transparent data management in a systematic manner, ${ }^{25}$ and was most suited to building on our previous research. ${ }^{1}$ The initial framework was informed by the literature, then tested and grounded in the data derived from the study subjects based on the accounts and observations of participants. In doing so, we drew on systems theory, ${ }^{28}$ involving macro-, meso- and microlevel factors, which have been shown to have wider relevance within dentistry. ${ }^{28}$

NVivo and Microsoft Excel were employed as tools for organising the qualitative data, facilitating analysis, including comparisons and associations within (and between) cases. ${ }^{25}$ Dual coding of the data was employed (FCS and JEG), with differences resolved by discussion. Quality was achieved and reported in line with COREQ standards. ${ }^{29}$

Analysis commenced with familiarisation with the data, which involved reading and 
Fig. 1 Provides an overview of macro-, meso- and micro-level contemporary factors influencing dentists' health and wellbeing. Micro $=$ personal, professional and social relationships. Meso $=$ job specification and workplace characteristics. Macro $=$ dental healthcare systems, regulation, profession and society. Adapted from Fiorella B. Colonio Salazar et al., 'Key determinants of health and wellbeing of dentists within the UK: a rapid review of over two decades of research' British Dental Journal, 2019, Springer Nature' and inspired by the Dahlgren \& Whitehead (1991) rainbow model of health and wellbeing

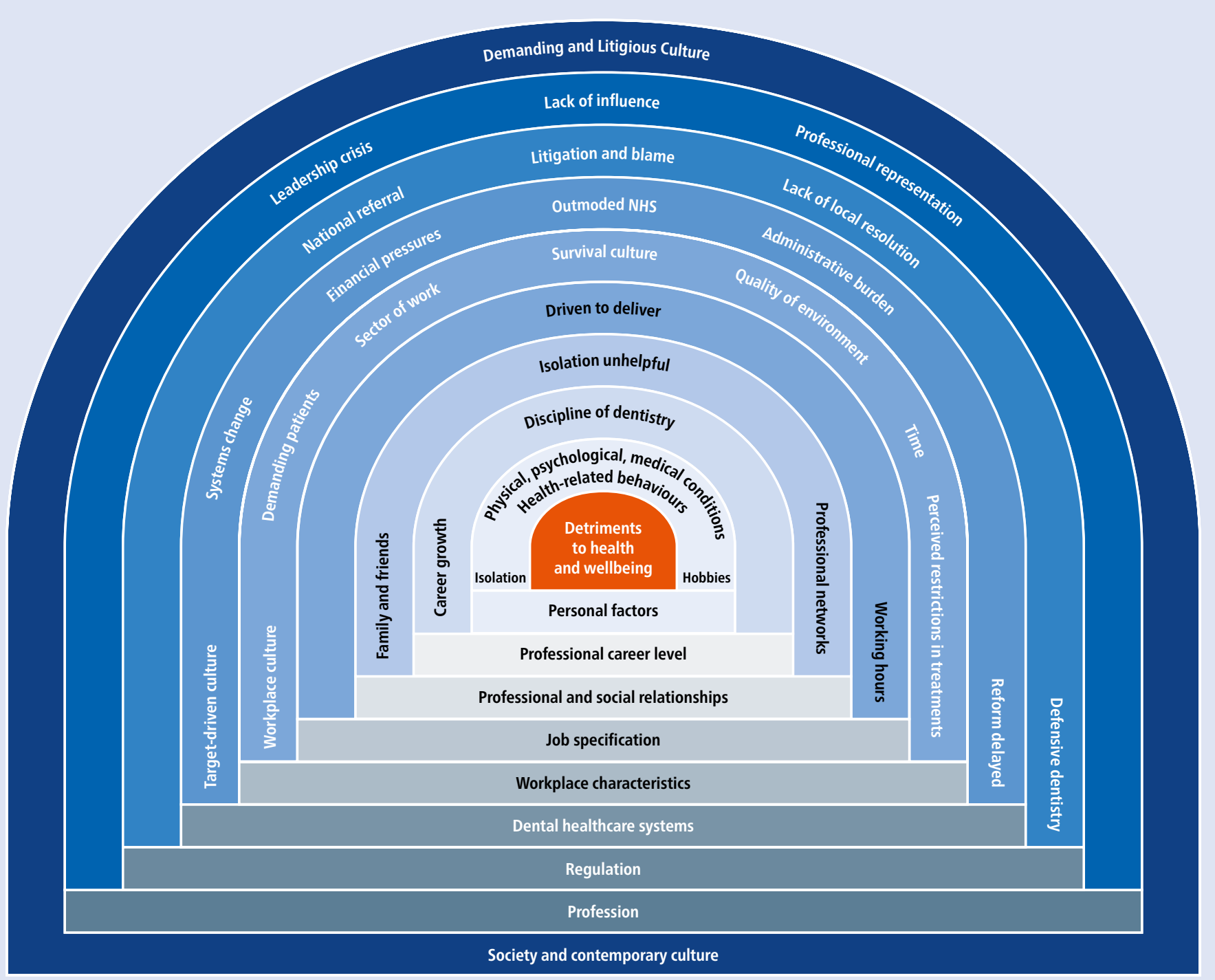

re-reading the transcripts and refining the initial thematic framework to ensure it was 'grounded in' (that is, reflected) the data collected. This was followed by application of the framework to the data, indexing and sorting the themes, reviewing quotes (data extracts) and leading to a descriptive summary which represented participants' views. Our analysis, taking an 'interpretative phenomenological approach', explored the stories (lived experiences) of participants. ${ }^{30}$ We produced a description of the themes (categories) and mapped them to allow explanation of the data and to build emerging theory which describes what is 'going on' in the data beyond mere facts. ${ }^{25}$

\section{Results}

Characteristics of participants

Twenty participants were interviewed, all of whom consented to participate and the majority of whom were women, qualified in the UK, at an early-career stage, working in primary dental care settings, holding mixed roles and based in London, as shown in Table 1. While five other dentists provided consent to be interviewed, three cancelled without providing any reason and two withdrew because of illness, choosing not to reschedule. Participants talked freely about their experiences and those of their colleagues.
Influences on dentists' health and wellbeing

A wide range of factors reportedly influenced dentists' health and wellbeing, as shown in Figure 1. Each one is reported in turn, starting with macro-level factors relating to professional regulation and system(s), through to meso-level influences including workplace and job specification, and finally micro-level involving relationships, professional careers and personal factors.

Quotations are used to illustrate the main findings. Each quotation is followed by participant demographic information relating to gender summarised as $\mathrm{M}$ (male) or $\mathrm{F}$ (female), the nature of their role, followed 
by the dental setting/service where they worked and interview number, limiting the descriptors for the purposes of anonymity. Current status of the dentist involved the following, recognising that some held more than one role: GDP (which may cover NHS and/or private practitioners); Principal; Associate; DO (Dental Officer); DCT (Dental Core Trainee); DFT (Dental Foundation Trainee); $\mathrm{T}$ (Teacher); SpT (Speciality Trainee); SP (Specialist). Descriptors also included the nature of their current service(s): CDS (community dental service); GDS (NHS general dental practice); Mixed (GDS and private); Private; HDS (hospital dental service); Acad (academia). All participants provided written consent to publication of this data.

\section{Macro-level factors}

\section{Regulation}

Concerns focused on the impact of current regulation on dentists' health and wellbeing in response to demanding and litigious patients. There was universal agreement that increased scrutiny from the GDC, to whom patients can complain directly, and the Care Quality Commission (CQC), which monitors standards across all dental services, were sources of stress and pressure for everyone. This was especially evident among NHS associates (dentists who work in, but do not own, the practice) and those in the early stage of their professional careers:

- ' the GDC comes down very heavily on GDPs or all dentists who make mistakes. I mean there is always this risk of you being reported to the GDC for even the minutest problem and then the GDC taking a worse decision on your conduct and striking you off from the list. So, you're under tremendous pressure all the time' (M-GDP\&T-GDS\&Acad[Int3])

- 'When the CQC came out as well, it was hugely stressful because we all had our systems in place and now we were [going to] get inspected by a body of [...] people who are not even in the profession, who will be holding us to a standard which is not designed for our profession. I remember spending many, many late nights doing that [...] It's very discouraging. It's demoralising' (M-PrincipalGDP-Mixed[Int13]).

There was a sense that recent experiences, including those of colleagues, had created a sense of fear of the GDC, leading to significant stress and demotivation:
- 'I think almost all dentists feel as if the GDC is kind of out to get us and they're standing against us... and I know they're there for the patient' (F-DCT-HDS[Int18])

- 'one is the transparency...there is no clarity as to how they [the GDC] arrived at that decision and who [was] involved in that decision. I'll tell you [...] a patient made a complaint against this dentist straight to the GDC [...] there was a letter sent from the GDC saying that you are under investigation for this [...] They took about nine months to investigate [...] It was only after many, many months we chased that [...] they came back and said, "Oh, by the way, the case is closed. There's nothing to answer." But the amount of stress that person [colleague] went through for that nine months, you know. And there was no apology, nothing at all [...] So, dentists are fearful of any letter from the GDC' (M-DO\&Associate-CDS[Int17]).

Dentists reported anxiety about possible complaints and litigation, with rising patient expectation/demands together with the current regulatory system, leading to a growing culture of 'defensiveness' and 'self-preservation' within NHS general dental services in particular. This reportedly resulted in 'defensive treatment planning, which put both dentists and patients at risk, as outlined below:

- 'You've also got patient expectations, nowadays in society I think we've got sort of an attitudinal shift where we believe there's always someone to blame; you know there's no such thing as an accident, and that's been fuelled by the legal profession in many ways where they promote litigation and that you have a right to redress' (M-GDP\&T-Mixed\&Acad[Int20])

- 'So, the entrepreneurial side of you gets eroded [...] and you could argue probably even deskilled because of that because you won't try new things, if you try something which is a little bit outside of your capabilities and you get it wrong you'll be criticised for even trying it and that's where the regulatory aspect of it become burdensome' (M-AssociateGDP-Mixed[Int8]).

One participant reported even feeling this on a daily basis:

- '[...] living in fear that this could be the day that someone reported you' (F-AssociateGDP\&FormerPrincipalGDS[Int15]).
Furthermore, most participants perceived that they spend longer than before on administrative tasks such as writing clinical records in order to 'cover their backs', given the increasing litigious environment, as suggested by this senior dentist:

- '[...] increasingly I see my young associates and dentists spending hours at the end of the day checking, going through the notes, you know spending their lunchtimes catching up with notes and letters and everything else. So, we spend more and more of our time doing the administrative side and making sure that our defence, should it ever be questioned in court or at the GDC, is robust and the way we wrote notes, the way we introduce things to patients again is increasingly defensive' (M-GDP\&T-Mixed\&Acad[Int20]).

\section{Dental healthcare systems}

\section{Target-driven culture}

Dentists currently inside, and those with former experience of, the system expressed strong discontent with the design and structure of the NHS dental contract in England, in place since 2006. Interestingly, senior staff were concerned about the pressures on earlycareer and frontline NHS dentists, regarding a system which was perceived to be primarily 'target-managed' and 'business-focused' rather than 'patient-centred'. Most associate dentists reported working under tremendous pressure when delivering care and this was confirmed by others, including senior colleagues. They reported feeling thwarted by the highervolume targets that they had to achieve within a limited timeframe in order to meet practice and personal financial commitments, with punitive sanctions (clawbacks) where they failed to do so and 'working for free' should they 'over-perform'. These were considered as major contributors to their demoralisation, demotivation, dissatisfaction and resulting stress, as outlined below:

- 'I think that's a significant factor [...] that dentists within the current NHS contract have punitive penalties if they don't hit their UDA target so they're having to work faster and faster and faster and they're being encouraged to deliver UDA-centred care so it's all about the UDA, it's not about the patient' (M-GDP\&T-Mixed\&Acad[Int20])

- '[...] but I still find the stress of the UDAs and...the pressure on associates around me, made me realise that I don't think I could do that long term as [...] my full-time job' (F-DCT-HDS[Int18]). 
Challenges in meeting targets at practice level included the practice principal's inability to cover staff maternity leave, together with other pressures which lowered productivity, as shown by the following quotation from more than one principal dentist:

- 'But my role as a principal I've got a target which is several different factors. If one dentist goes off on maternity leave and another dentist is [...] sick, or another dentist is performing poorly, and the target is not being reached, I can feel that pressure because [...] there are financial penalties to not achieving that' (M-PrincipalGDP-Mixed[Int13]).

One mid-career stage practitioner reported selling his practice because, although he supported his female dentists' periods of maternity leave, the pressure of managing the NHS contract and engaging appropriate levels of cover was a logistical nightmare and left him 'burnt out'.

\section{Financial pressures}

Concerns regarding the profitability and sustainability of NHS general dental services in the future, exacerbated by the uncertainties over Brexit and lack of progress with a reformed dental contract, were mainly reported by principal dentists. Dentists reported that NHS dental contract payments had remained the same for a long period of time, whereas the cost of running dental services had significantly increased and resources were being lost from NHS dentistry through financial 'clawback' when services couldn't be delivered in-year. In addition, practice principals from outside London reported that recruitment of associate dentists to deliver the contract was difficult. The ensuing financial pressures were a major source of stress, as illustrated below:

- 'So, everyone's paying more but as private fees, I can adjust them to reflect that... But with the NHS fees, we can't...And instead of the NHS England doing something about that they just leave it. Which means that we're getting poorer every year which is unsustainable. Eventually you'll reach a point where it just doesn't actually serve your economic interest to provide NHS work at the costs which you're providing it at' (M-PrincipalGDP-Mixed[Int13]).

\section{Systems change}

Some participants working in community dental services appeared to be facing challenges resulting from recommissioning of NHS service contracts, as well as internal restructuring where, in addition to multiple service and professional demands, the threat of possible job losses loomed large:

- '[...] we had a restructure [...] I think it was around April last year (following recommissioning) where we were told that there was going to be some job losses within our service. So, the whole process was quite unsettling to be honest... and then we had [...] not enough funding to have a receptionist at our clinic anymore...then also most of our nurses who are at a band five level were demoted to band four. So quite a lot of the staff felt quite demoralised to be honest, and a couple of my long-term colleagues got made redundant...our Assistant Clinical Director, she just found the whole thing quite stressful and she left as well' (F-DO-CDS[Int16]).

Overall, there was the recognition that contemporary care involved managing ethical dilemmas on a day-to-day basis, with implications for dentists' health and wellbeing.

\section{Professional leadership}

In response to the above issues, there was some concern that professional leadership did not reflect the demography of the profession and that younger dentists were not being engaged enough for their views on the future of dentistry.

\section{Meso-level factors}

\section{Workplace characteristics}

Participants suggested that the sector of employment, together with the culture and quality of their working environment, influence dentists' health and wellbeing.

\section{Workplace culture}

Most dentists reported that NHS associates were immersed in a 'culture of survival' within general dental practices, in which self-preservation was paramount in response to growing macro-level influences outlined above. This was resulting from the constant pressure within their organisation to protect themselves from litigation, as well as to achieve NHS contract targets and retain their jobs. This culture appears to be challenging dentists' principles, in which 'cutting corners' to achieve targets and practising 'pragmatic' or 'defensive' dentistry were becoming accepted practices. Examples ranged from staging treatment plans to withholding prevention, thus failing to place patients at the centre of care, as outlined below:
- '[... you don't get paid anymore whether you do the fluoride varnish, or don't you know. So, lots of dentists don't seem to bother doing it, well [...] it's wrong of the dentist to not follow the guidance [...] even if the NHS aren't giving you anymore money you should still do it because it's the best thing to do but the NHS system doesn't encourage good behaviour if you know what I mean' (M-DFT-GDS[Int9])

- '[...] There are lots of cutting corners and I don't think the practice was safe for patients or clinicians...I wasn't happy to practise in that environment...I'll give you a simple example which, basically, on my first day I had root canal treatment and they didn't have a rubber dam kit. They said it's a non-surgery practice. They have one rubber dam kit that gets used by the foundation trainee. I was like, "Well, I want that kit". And I'm not happy to carry it out without a rubber dam. So yeah, general practice is very business-orientated and moneyorientated' (F-DO-CDS[Int19]).

Managing ethical dilemmas, as a result of very real tensions over quality standards of care possible in NHS general dental services, was identified as a constant stressor:

- 'One of the dentists in our practice was behind on their target and the boss just simply said if you don't hit this number you're out and so when you see that you think well I don't want to do it then because I don't want to be in that situation where if I do the thing that, if I follow the best evidence I will lose my job' (M-DFT-GDS[Int9]).

However, the pressure of family financial commitments meant that some were unable to take the risk of just walking away from NHS dentistry, as suggested by the following quotation: - '[... if I was given an alternative... if I had an opportunity to quit working as a NHS general dental practitioner I would do it any day, I would leave it completely; but I can't because I have a family to bring up, I have children going to school, I have a mortgage to pay, so I can't stop doing that' (M-GDP\&T-GDS\&Acad[Int3]).

\section{Sector of work}

Opportunities for continuing professional development differed across the sectors where dentists worked. Those working for NHS general dental services repeatedly reported their dissatisfaction and demotivation, and were keen to upskill or progress within this sector. For 
most, undertaking routine and simple dental treatments in primary dental care failed to challenge them intellectually and clinically and provide professional fulfilment. Additionally, early-career staff raised concerns that some senior colleagues in general practice did not maintain their knowledge base over time:

- 'There's quite a lot of people who are not up to date in their dentistry [...] aren't aware of guidelines, and just kind of practise the way they've been practising for a while' (F-DCT-HDS[Int18])

- 'In the same four walls, with the same people you have to work with. Some people like that, they like the comfort, fine, but the challenge of clinical work, yes, it's a challenge, but if that's my only challenge, and I hate doing it, I don't get to challenge my brain' (F-DFT-GDS[Int12])

- '[The practice boss said] "I'll do consultations down here. I'll do the surgery up at the specialist centre. And then I will show you guys how to restore the implants, and then you can start to restore the implants yourselves and upskill". I said "Great, this is fantastic". It hasn't materialised yet; I haven't given up hope on it coming through. But that was a big boost when I heard about that; I thought, "That's great, a real opportunity to actually upskill"'(M-AssociateGDP-Mixed[Int10]).

Working for others, particularly for corporate bodies, was perceived to limit dentists' options professionally, financially and clinically. Although there might be leadership from a practice manager, this was not a replacement for the professional mentoring support that might be available in a traditional practice where there could be face-to-face access to clinical leaders on clinical issues:

- 'The corporate is that there is no one to go to, they'rae all equal associates here [...] the practice manager is not someone you can go to on a clinical basis [...] you need to see someone face to face and on the end of the telephone is not really ideal' (F-GDP-Mixed[Int7])

- 'But when I did the corporate [dental practice] it was a lot about the money again, that came back again, especially that they have targets. They put your name on a board in the staff room, and you are green if you've hit your target, you are amber if you've not so much, you are red if you haven't. That really frustrated me, because I thought, oh, gosh, here it is again, it's about the money. Which is fine, it's a business, it's okay, but
I think personally, in an NHS practice, I find that a really big stress factor, money' (F-SpT-HDS[Int11]).

While some would like to open and/or run their own practice, this was not a general view among participants:

- 'I can't buy into the practice because it's owned by a corporate' (F-GDP-Mixed[Int7]).

In marked contrast, dentists working in dental teaching hospitals reported enjoying teaching and learning opportunities and were positive about being stretched, as demonstrated by the following quotations:

- 'I like being in hospital [...] I like being around people who are doing research, trying to change the way things are [...] and also doing the top level of dentistry, being back [sic] what you were taught in dental school...I think it's much more interesting, you see a lot more diverse cases, it's much less monotonous as well' (F-DCT-HDS[Int18])

- 'The other side of working in a hospital, which I enjoy [...] I became a consultant to have that interaction with the trainees, and be able to [...] supervise, guide them, teach them, that sort of thing [...] which I find satisfying because it gives you a bit more diversity in terms of your job' (F-SP-HDS\&Private\&Acad[Int6]).

\section{Quality of working environment}

Dentists' quality of working environment was fundamental to their professional satisfaction and resulted in the following dentist withdrawing from delivering NHS care. In relation to potential litigation from patients, NHS associate dentists' frustrations were exacerbated by feeling more vulnerable and unprotected in their workplace compared with their salaried colleagues working in larger organisations, as stated below:

- 'It's a really horrible profession to be in now, from the point of view that no one stands up for you. That's why people want to work in hospital, because they have a huge body behind them who will help them in a difficult spot. Whereas in [dental] practice there is really no one there for you, standing up for you' (F-DFT-GDS[Int12]).

\section{Lack of material resources}

Participants from NHS general and community dental services reported feeling frustrated due to the lack of material resources limiting the opportunity to meet higher and more challenging targets and putting at risk the quality and standards of care, as suggested below:
- In terms of the equipment [...] as well, like when lots of treatment is booked you find that you run out of certain essentials erm, when there's no scaler... The clinic runs on three handpieces and if they run them through after each patient...you just don't have the material and the equipment to do what you're meant to do' (F-DO-CDS[Int19]).

\section{Patient demands}

Handling very demanding, frustrated, aggressive and unhappy patients, with apparently high and unrealistic expectations, was reported as a common stressor among participants across services: mixed, private and NHS general dental services. Furthermore, action taken by patients discontent with any dental experience was a source of real concern, linking to professional regulation, as outlined below:

- '[...] we're far more accessible. People can verbalise their views, whether it's a Google review or a whatever review or they can e-mail the practice and you are accountable and you've got to be pretty on your toes to respond to their dissatisfaction and it's becoming a lot more easy [to complain]' (F-SP\&T-Private\&Acad[Int5])

- '[...] he's upset because the treatment has been dragged out for so long but obviously, it's an NHS waiting list so that's why it's taken a long time. And he's going to go and complain to the MP about me because he doesn't believe that he's having the correct treatment, even though weve been doing so much treatment on him and sending off so many claims. I could get flagged up for it...So that kind of thing runs through your head as well; are you going to get sued...is your reputation going to be tarnished if you get flagged up for things with the Dental Board [NHS payments agency]?' (F-GDP-Mixed[Int7]).

\section{Time pressures}

Working constantly under time pressure to see a large volume of patients per day and/ or completing administrative tasks outside of contracted hours, as well as the challenges of IT systems and programmes, were sources of stress and job dissatisfaction among dentists in most dental settings. Hospital services were not without their pressures too, as illustrated by the following quotation from a consultant:

- 'Too many patients, too many things going on at the same time. I mean ideally if I've five patients in the afternoon, okay I keep hearing my colleagues saying you can't see more than two of those in an afternoon...If 
I have six [...] maybe seven or eight like I had yesterday...I'm running, running, running, running. And I know because when I've had a day like that I go and take my blood pressure and find it's up a bit, but I go home. So that's stress. That's not really what I want to be doing, but you've no choice when you've got that number of patients. So that's why I get stressed' (F-SP-HDS[Int2]).

\section{Staffing and quality}

Understaffing, resulting in dentists having to increase their workload to keep the system running, was a concern as it impacted on clinical care. Requirements to take over reception duties and certain nursing roles appeared to affect community dentists in particular and those working outside of London. Doing so had implications for patient flow and was a source of frustration. Examples of feeling overwhelmed, dissatisfied, demoralised and stressed are provided below:

- 'We don't have admin support to do that [administrative tasks] for us...I mean the NHS in general is understaffed and under-resourced and on top of that they want results and I guess like you don't have a clear mind erm, because of the work pressure. You come back home, and you want to switch but sometimes that's difficult...I think my family noticed that I'm always thinking about work and what to do and it can make me upset' (F-DO-CDS[Int19])

- 'So, whereas before, stock control was nothing to do with me, not under my remit...I'm now involved in that and I'm chasing things, and it's taking my time away from what I should really be doing...there's just not the people on the ground to do it...just the lack of staffing and the fact that I think the people who were doing their jobs are now being given so much extra to do that people are just overwhelmed really' (F-DO-CDS[Int16]).

\section{Ongoing change}

Change, including staff turnover and new ways of working, was a source of frustration and stress among dentists, including private practice - 'things are always changing' (M-MixedGDP[Int10]):

- '[...] it was difficult at first cause I used to work with the same nurse who knew all the orthodontic instruments and all the rest and then, all of a sudden, she left...I then had somebody who had, you know, absolutely no clue what was happening but it's just, you know I asked for a certain instrument [...] and they go, "what?" It's like, it's fine...show me the box of instruments so I don't have to de-glove' (F-SP-HDS\&Private\&Acad[Int6]).

\section{Job specification}

Most dentists working in primary care are selfemployed; however, these terms of engagement, which historically were beneficial, are currently viewed by some as less favourable for health and wellbeing. There were clear implications for the health and wellbeing of salaried and self-employed dentists, as reported below. It appeared that self-employment and lack of permanent contracts were major stressors among those who had experienced these challenges. Frustrations were expressed during interviews, with participants discussing alternative routes to find occupational stability:

- 'There's no appreciation from [management] to the work you do [...] As well as that, I did mention that I'm still [...] on the bank [temporary staff]. So, I don't have a substantive post...my long-term plan is to specialise in dentistry...Yeah, I'm looking for vacancies to apply [...] maybe with different services...there's a lot of stress added on' (F-DO-CDS[Int19]).

One practical example relating to selfemployment involved 'annual leave'. Associate dentists appeared not to take their full annual leave allowance in order to deliver their NHS targets, recognising their financial commitments (family and practice), thus contributing to their exhaustion and stress:

- '[...] the downside is that because you're still classed as self-employed there is no holiday pay, so when you're not working you're not earning...So, the balance of taking all your time off is great for your time off but of course you're not going to earn any money to pay the bills if you're not in work' (F-DCT-HDS[Int18])

- 'I mean I've seen one of the associates that I work with at the moment on the UDA contract just hasn't taken a holiday in the whole time I've been there, and I just think what a horrendous thing to be, that's the same one who is behind on his target he doesn't take any time off and I just think it's so sad' (M-DFT-GDS[Int9]).

In contrast, early-career dentists without significant commitments or dependants reported that while their income may be lower, their preference for having a job they enjoyed which enabled them to be happy and healthy led to their decision to remain salaried, as indicated below:
- 'I mean I get paid holidays, and I get sick leave, I even get compassionate leave and carers leave and all these different things, and fixed hours [...] then I think for what I do compared to what I did when I was a GDP [income is fair]' (F-DO-CDS[Int16])

- 'Because I don't have anything that I need the income for, I mean obviously I need to pay rent and I need to pay travel...I don't have any children, I don't have anything that I need to be paying for, I don't have a mortgage. So, it's not a huge thing for me...so I prefer to do something that I enjoy, so be in hospital, and to feel happier about my job than being an associate and earning more money at the moment' (F-DCT-HDS[Int18]).

\section{Micro-level factors}

Personal, social and professional relationships

Absence of protective relationships was a perceived source of stress. Physical proximity to family and friends was particularly relevant for early-career dentists in salaried and non-salaried posts to feel reassured and re-energised. For some, working away from home produced a sense of loneliness and isolation, which was extremely stressful. Dentists commented on the efforts to visit their close family and friends to maintain positive relationships and feel invigorated, as stated below: - 'I think I underestimated the lack of support system. I moved to this new place, into a house with someone I didn't know, to work in a practice with people I would have to get to know over the year. But none of my closest friends were there, and I spent most of my weekends organising to go away to see them, and that obviously takes a lot of time out as well' (F-DFT-GDS[Int12]).

In contrast, members of the profession with strong family and social relationships reported being in a much better place in relation to their health and wellbeing. This was particularly apparent where dentists had a strong meaningful relationship with a stable partner.

\section{Professional relationships}

Differences emerged between dentists working in NHS general dental services, including corporate bodies, and those working in hospital, community and private practices in relation to the nature of their relationships with workplace colleagues. NHS general dental practice was perceived as more isolating on a day-to-day basis, with little or no peer support: 
- '[...] whereas in general practice, everyone is working really hard, no one really has time to help you, no one has time to answer a question, they are all trying to do all of their things all at once as well. Equally they are self-employed, so any time they don't spend with patients they are not earning money. Whereas being salaried you have the luxury of asking someone a question, going back to the patient, over-running 20 minutes, or the next patient doesn't come but you still get paid regardless' (F-DFT-GDS[Int12])

- ' [...] the corporate mentality is that there is no one to go to, they're all equal associates... certainly in 'corporates' you do get very isolating erm associates who don't like talking to others, they will just stick to their own' (M-AssociateGDP-Mixed[Int8]).

While some organisations were perceived as settings in which participants were 'just a worker' and not 'valued', other practices which tended to be larger and mixed, or mainly private, provided a positive professional environment in which dentists felt 'valued' and cared for:

- '[...] Not in general practice, and the boss, again, introduced himself with a very hilarious statement where he said no one is going to give you a gold star for doing it well, you just do it well and you move on' (F-DFT-GDS[Int12])

- '[...] that's the patients and the staff, the team that I work with or as part of that's where I get my enjoyment and professional fulfilment particularly young dentists coming through. So just running cases by each other and whether it's having a bit of a laugh at lunchtime or a shoulder to cry on in the evening' (M-GDP\&T-Mixed\&Acad[Int20]).

Participants, notably hospital, community and private dentists, reported how their positive professional relationships with colleagues and patients supported by teamwork can lead to a healthy and supporting working environment, keeping dentists motivated and satisfied. Moreover, working with the same colleagues was perceived to enhance collaboration and morale, as reported below, across settings:

- '[...] so, I think working in practice mainly it's the patients, and the members of staff around it. If they make it, you know, it's a nice environment, you know, you feel looked after, you look after the patients... you just create that happy atmosphere' (F-SP-HDS\&Private\&Acad[Int6])
- I'm now working, more or less, with the same sort of nurses, so that's been good and so there's more team spirit I think'(F-DO-CDS[Int16]).

\section{Professional career level and stage}

Dentists discussed how their stage of professional career influenced their health and wellbeing. Among middle- and late-career dentists, there was an apparent confidence in undertaking their daily clinical and non-clinical work, reporting positive morale. Later-career dentists generally associated with increased confidence in their knowledge and skills, but sometimes growing frustrations. Early-career dentists reported striving to build their confidence, skills and knowledge, including their knowledge of the wider health system, together with high aspirations in relation to quality:

- '[...] because I've done such a volume of that in the past, you know...it's helped me to keep my confidence really...I do like to go on courses and keep up to date' (F-DO-CDS[Int16]).

However, they expressed their fear of the unknown and concerns about making mistakes. Doing 'things right' and at a 'high standard' was commonly discussed among these dentists. Limited opportunities for building confidence within their workplace were a source of frustration, as indicated below: - 'I like it [emergency care] because it's acute care...I worked in the hospitals before so [...] I feel like my skills are mostly in like, emergency care...Also, I was pushing for the training...you need pharmacological intervention sometimes...I have been on like, restorative courses to improve my clinical skills' (F-DO-CDS[Int19])

- 'It makes you feel like you can't do it, and then I'm like if I can't do it then why is he not telling me. Or why is he, or she, not saying, stop for a moment, you do it, and let me just talk you through it really slowly, and then you will learn how to do it. Something like that is better than saying let me take over, because if that person takes over, and you don't use your hands, then there's no point' (F-SpT-HDS[Int11]).

Uncertainty among early-career dentists over future plans and opportunities for career progression, including specialisation, were ongoing concerns, as demonstrated by one core trainee who decided to leave NHS general dental services:

- I would say the biggest concern I have is what I'm going to do after this year and where my career is going to go, because at the moment
I am just doing kind of year post, last year I had no idea what I was going to do at the end of the year, this year again, it's just a year and what am I going to do next [...] and how am I going to increase my skills or stand above the rest of the people applying for the next hospital job? So, I would say that's my biggest concern at the moment' (F-DCT-HDS[Int18]).

\section{Personal factors}

In relation to personal health and wellbeing, participants referred to physical, emotional and psychological dimensions of health, and a wide range of perspectives emerged. Several suggested that their perfectionist and highachieving personalities were a source of stress in different roles and settings. In terms of physical health, all late-career dentists interviewed reported enjoying a good lifestyle and health, and while they acknowledged having physical or medical conditions, these were considered 'normal' for their stage in life. Dentists working in salaried and private practice shared similar views, relating any conditions to the hazards of their practical work. Physical conditions were a matter of greater concern for early-career dentists working in different settings due to their long-term career implications. Some had sought various therapies to assist with practical challenges such as back pain:

- 'I've been having quite a lot of back problems recently [...] and I had one incident where I actually didn't go to work because [...] it was so bad in terms of stiffness and pain where I couldn't actually move my neck for [...] a little bit...I'm still obviously very young, so I still have a lot of [...] working days ahead of me and I don't want this to become an issue in the long term [...] It's something that I was not even thinking about before' (F-SP-HDS\&Private\&Acad[Int6]).

With regards to emotional and psychological health, most associate dentists holding significant NHS commitments, including early-career dentists in primary care, reported feeling unhappy, anxious and stressed. Some had personally been diagnosed with depression and were taking medication at the time of their interview, or reported low morale and acknowledged having had suicidal thoughts in the past. There was awareness too of dentists who had committed suicide. Nonetheless, there was evidence of these participating dentists seeking counselling and support:

- 'So, after Istarted working as a GDPfor National Health Service, I have been on anti-depressants 
for almost four years now... think this pressure was threatening to come to a point where I was getting more and more depressed...I'm under the care of a psychiatrist right now' (M-GDP\&T-GDS\&Acad[Int3])

- 'I was trying to do good dentistry, but I wasn't making targets. So as far as the practice was concerned, I wasn't necessarily a "good" dentist [implying not earning enough]. So, I did feel a little bit undervalued. I was feeling a bit [sic] low self-esteem, just generally miserable and often quite tired. I have had suicidal thoughts before, but [...] never went near any risk of harming myself, so that was alright' (M-GDP-Mixed[Int10]).

Others shared their stories, reporting feeling stressed, experiencing symptoms such as 'ringing in their ears' or were in a position where they 'broke down in tears' having 'had enough. There was a recognition that personal issues such as relationship breakdown and bereavement compounded the situation where 'work' affected them. At these times, support from family members and partners was strongly acknowledged as important.

When health-related behaviours were explored, most participants reported adopting positive health-related behaviours such as physically exercising and consuming a healthy diet. Those who were diagnosed with depression tended to describe episodes of heavy regular alcohol consumption and disturbed sleep patterns, while others acknowledged heavy work commitments interfered with aspirations of a positive lifestyle where sport, music and other restorative elements had been squeezed out by professional life:

- '[...] my personal life was getting quite irregular [...] my wife was beginning to tell me that I was very moody, and I used to snap at very small things. I had become very snappy at my place of work. I think I was quite angry all of the time and I started drinking quite a lot' (M-PrincipalGDP-Mixed[Int13])

- 'My sleep is not very good. Sometimes my work/life balance is not quite what I would like...I wonder if some of it is money worries... It's nothing too bad, but I had a couple of months off in the start of the year because I broke [bone]...The car broke during that time as well, so I had to pay for that [...] That's one little bit of stress, I guess, which is sorting money out' (M-GDP-Mixed[Int10])

- '[... I used to play a lot of sport and I used to coach rugby up until about 10, 12 years ago but that just sort of got squeezed out because of other commitments to do with dentistry' (M-GDP\&T-Mixed\&Acad[Int20]).

When dentists were asked about influences on motivation and job satisfaction, altruism and passion for their profession were commonly reported. Making a difference to patients in need, and their organisations, was considered rewarding and fulfilling, and part of the stress was related to the fact that they cared about being able to care for patients through dentistry:

- 'I think in my job when working with children who are coming in too anxious, nervous and the worst-case scenarios when they've had a bad experience [...] and you actually manage to turn someone's experience of dentistry around and they actually leave the surgery smiling, and, and clinically you managed to get something done, it's actually quite difficult, I mean try seeing a four-year-old and taking out six teeth, and have them still laugh about it you know [...] that side of it, I find that really rewarding [...] just meeting that challenge really of getting the work done, when no one else could, erm especially being in a referral service, someone's already tried to see that patient and failed and so when you have that success it's quite satisfying to be honest' (F-DO-CDS[Int16]).

Dentists reported being energised by being able to deliver holistic, patient-centred, highquality care:

- 'I actually enjoy the dentistry part of things, I enjoy doing it, I find it really interesting [...] and I do also want to make people's oral health better [...] I wouldn't really say that money drives me too much in hospital, because you don't get paid very well in hospital...but it's more kind of making a difference to patients...that kind of [thing] motivates me...you could sometimes feel that you were making a difference in this patient and I saw one patient who was homeless before and he moved into kind of sheltered

Fig. 2 Emerging contrasting themes on dentists' health and wellbeing. Note: early-career dentists and frontline NHS practitioners were generally perceived to be focused in the 'red' zone and senior colleagues in the 'green' zone

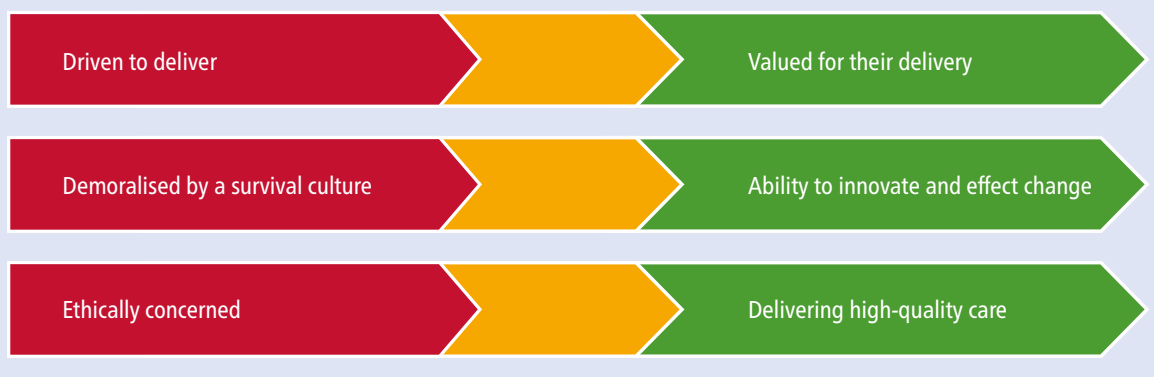

accommodation [...] and I saw him from the beginning of his treatment and kind of stabilised him. We got him a set of dentures and [...] then towards the end, he was like, "I'm going to job applications, I feel so much better about myself". So, there was occasionally these big cases, where you were like, you've actually made a difference in these people' (F-DCT-HDS[Int18]).

Making a difference within organisations was also energising and rewarding. Changes implemented and resulting in positive outcomes kept mid- and late-career dentists satisfied and happy:

- 'I would say I'm in a position where I can see some of the changes we introduce, and a few months down the line we can see what the results are. I think that's very important... seeing that you're making a difference...we're getting some feedback that different things are becoming more active...it's from both staff and the patients...So I think it's fortunate' (M-DO\&Associate-CDS[Int17]).

Participants also reported how valued and recognised they felt at work by patients and the team (horizontally and vertically) which contributed to their morale, motivation and job satisfaction including tangible demonstration of their satisfaction, as described below:

- '[...] really nice comments from them [patients] saying that they're very satisfied. They want to stay with you...I have [...] presents from children. They come with drawings or something [...] which make you happy. Gifts from patients [...] because they're satisfied' (F-DO-CDS[Int19]).

\section{Emerging theory}

Three important contemporary themes emerged for frontline NHS practitioners, as presented in Figure 2. Participants reported either currently or previously experiencing these challenges or having witnessed others facing such challenges. 
First, frontline dentists, particularly juniors, were driven to deliver under the influence of the 'NHS national dental contract' and its implementation within the workplace culture of general dental practice, where the business of dentistry was perceived to be prioritised over patient care and fuelled by their personal financial aspirations or requirements. Second, many were ethically concerned as they moved from the supportive context of a dental school to work within NHS general dental practice where there was little support for delivery of patient-centred and evidence-based care. Third, participants felt they were demoralised by a survival culture influenced by the workplace characteristics and their job demands, where there was little opportunity for development. This was compounded by regulatory and systems factors, in a system and practices that placed business before care and a situation in which they were often just workers. Personal and social protective factors were considered insufficient to counteract these pressures; this resulted in perceived lack of control overall and impacted professional fulfilment in dentists, with implications for their health and wellbeing, particularly involving those at the NHS coalface. This was in marked contrast to senior dentists, who more often reported using their expertise, having risen to roles where they were using their highly refined skills, in systems where they were valued for their delivery. There was a strong emphasis on team effort with a collaborative ethos in delivering quality care and the ability to innovate and effect change within organisations for the benefit of patients and staff through direct patient care.

\section{Discussion}

This research provides important insights into the health and wellbeing of the dental profession, drawing on the experiences of dentists in England. Our results amplify the findings of recent national surveys, ${ }^{6,7,8,9,10,26}$ and the range of macro-, meso- and microlevel influences identified in the literature, ${ }^{1}$ which in combination affect dentists' health and wellbeing, as shown in Figure 1.

Evidence from this study confirms macro-level challenges including regulatory

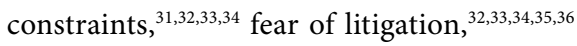
financial pressures, ${ }^{35,37}$ target-driven restrictive NHS contracts ${ }^{9,10}$ and professional leadership. ${ }^{4,7,27}$ Similarly, meso-level challenges such as: reduced opportunities for career progression, ${ }^{23}$ particularly for those in contracted positions within the NHS; ${ }^{38}$ increasing time constraints; regulatory obligations for practice owners; $;^{39,40}$ and professional isolation, ${ }^{9,36}$ are reinforced. Emerging meso-level limitations such as ethical dilemmas, financial constraints, lack of occupational stability and restrictive working arrangements in corporate organisations are also highlighted. It confirms known microlevel issues faced by dentists related to their physical, emotional and psychological health, as well as the influence of social and professional relationships, ${ }^{9,35,41}$ while also presenting emerging perspectives on dentists' personal motivation to deliver high-quality, patient-centred care.

Importantly, this research provides further evidence that macro, meso and micro influences often compound one another, as suggested by our rapid review. ${ }^{1}$ The problem of burnout and risk of suicide have long been recognised in dentistry, often as a result of multiple pressures. ${ }^{5,42,43} \mathrm{We}$ are not alone nationally or as a profession. Our findings confirm evidence emerging from dentists across the UK and beyond, ${ }^{44,45,46}$ together with our medical counterparts nationally and in other high-income countries. ${ }^{47,48,49,50,51}$ Such is the scale of the problem that it has been termed an emerging 'public health crisis. ${ }^{49}$ Interestingly, the National Academy of Medicine in the US, ${ }^{52}$ has developed a conceptual model which appears remarkably similar in its components to Figure 1. While the findings seem shocking, it is important that they are considered within the wider context of health services where clinician wellbeing is considered 'essential' for 'safe, high-quality patient care'.

Dentists are deeply discontent with the NHS primary care systems. Dental services, whether general or community, were perceived by most participants as being primarily 'businesscentred', with pressure to meet practice contracts and targets under the current NHS system and little room for manoeuvre. Many dentists personally reported working in organisations where the culture emphasised the business of dentistry over meeting patients' needs, in which junior dentists were driven to deliver working under tight controls, leaving these early-career dentists without the time to deliver evidence-based dentistry such as preventive care. Challenges include corporatisation of primary dental care, together with practice culture where associates are heavily managed, often by non-clinicians, in relation to their productivity. This engendered feelings of hopelessness and frustration. When compounded by personal isolation or physical problems, it begins to create a 'perfect storm'. Senior colleagues raised concerns over the challenges being faced by early-career dentists, legitimating their views. These findings help to explain the level of discontent with NHS dental care, with recent reports of $58 \%$ of NHS dentists reporting their intention to leave or scale down their commitment in the next five years, ${ }^{12}$ and $75 \%$ of practice owners struggling to fill vacancies. ${ }^{13}$

Moreover, we found evidence that principal dentists particularly experienced challenges resulting from concerns about the profitability and sustainability of their practices, particularly in relation to the NHS. It is not surprising that many of the profession have sold their practices to corporate bodies as they move towards retirement. The impact of the type of NHS contract on the business of dentistry, together with Brexit, was of great concern for principal dentists. Given the current political situation nationally, there is a need to actively support and nurture dentists, recognising the changing context including corporatisation, the importance of access, ${ }^{53}$ and the oral health needs of our ageing population..$^{54}$ As we finalise this paper for publication, the current COVID-19 pandemic, ${ }^{55}$ is providing an unwelcome disruption for most of our profession and patients; yet, this must be an opportunity for change to enable long-term improvements in morale, health and wellbeing, and to refresh our assets. Our findings suggest that participants perceived private practice to be less stressful and thus more supportive of health and wellbeing. While COVID-19 has presented new challenges for NHS dental services, ${ }^{56}$ it has also highlighted that private practice itself is not immune to challenges, when services generally are suspended and result in loss of earnings.

Although workplace characteristics, including understaffing and time pressure, are not new in the literature, ${ }^{57,58,59}$ our findings suggest that limited opportunities for career progression and diversification were sources of particular job and career dissatisfaction. ${ }^{23}$ Most dentists have traditionally worked in primary dental care, providing varying levels of NHS dental care under contract. Salaried positions whether in speciality, community or academic roles within dentistry have always been limited in number, as with opportunities for solely private practice. However, in the 
past, most dentists worked as an associate in an NHS dental practice for several years, consolidating their clinical skills and acquiring business acumen to enable them to set up their independent practice and control as well as manage it with their own distinctive approach. Such opportunities are now increasingly rare. Recent graduates, therefore, increasingly feel professionally constrained, as reflected in the participant responses discussed above. Furthermore, eligibility and affordability of financial loans to set up a practice are increasingly difficult given significant student fee debts, ${ }^{60}$ which further limits graduates' future possibilities. Health Education England is currently exploring professional careers in dentistry, enhancing flexibility into training pathways and providing career development opportunities as mechanisms to improve job satisfaction and encourage retention. ${ }^{23}$

In recent decades, dentistry, as with healthcare in general in England, has been aligned with a neoliberalist philosophy that sought to extend market mechanisms across the welfare state. ${ }^{61}$ There is now a move back towards the ethos and historical values of an NHS to re-embrace 'collaboration' and 'mutualism', and build a future based on collaboration. ${ }^{62}$ Given the challenges facing our profession, it would seem prudent to invest in radical reform at systems level and involve a wider societal debate. Furthermore, at organisation level, as with many large businesses, we should be working out how to engage younger generations in the workplace ${ }^{63}$ and ensure implementation of solutions that work beyond mere 'productivity'. Dental practices require clear clinical leadership, particularly where they are part of larger organisations. The next paper in this series will explore possible strategies and solutions in a whole systems approach.

On a more positive note, despite the challenges, dentists achieve great satisfaction from 'making a difference to patients', particularly those with the highest needs, and this contributed to their sense of professional wellbeing. Although anxious and demanding patients were mentioned, they were much less of an issue than expected. ${ }^{32}$ A significant part of dentists' frustration related to the fact that that they could not routinely provide high-quality contemporary care, practise ethically and gain sufficient financial reward to make the stress and risk worthwhile. Overall, however, dentists with close personal and good professional relationships, well-developed careers, clinical stability and expressing professional fulfilment tended to report better health and wellbeing. While these dentists recognised the negative aspects of their career, they had demonstrated the ability to work around the challenges they faced, often broadening their roles to find a niche with sufficient power and status to effect systems change, which may be termed resilience. ${ }^{48}$ While many may have learned through experience how to manage pressure, negotiate change and build resilience, and achieve more positive outcomes as presented in Figure 2, they also did so in a very different world which may no longer be realistic for the emerging workforce. This highlights the importance of supporting personal resilience in our younger colleagues which, although vitally important, is not the sole answer to our challenges.

Certain limitations of this research, however, must be acknowledged. First, it was pre-COVID-19, which has brought new stresses. ${ }^{56,64}$ Second, although purposive sampling was undertaken via gatekeepers nationally, dentists from London and the South East of England were overrepresented. The findings thus need to be treated with caution. The apparent differences between those working within and outside of London, as well as the improved resilience in senior dentists, will require further exploration. As we draw to a close, it may be helpful to recognise the purpose of qualitative research, which is to explore a range of perspectives and possible influences, and not to quantify or provide statistical generalisability; therefore, small well-designed samples are legitimate. Furthermore, with all the above limitations, the research findings merely amplify the findings of academic, ${ }^{1,4,5}$ and health service literature nationally. ${ }^{7,8}$ We trust that this paper will be useful to stimulate debate, inform future research and result in timely action to protect the workforce - a valuable asset. ${ }^{65}$

\section{Conclusion}

Evidence from this study confirms that dentists' health and wellbeing is affected by multiple contemporary influences and highlights challenges to be addressed. Therefore, we strongly recommend that as a profession, we take these issues seriously and consider potential solutions as a matter of urgency, particularly at systems and organisational level, together with ensuring greater support for individuals.
Acknowledgements

The authors would like to thank Public Health England for funding this project, key gatekeepers across the country for their support in the recruitment process and all the dentists who participated in this study. Our thanks also to Dr Jim Aukett, who provided advice on an early version of this manuscript, and Sukriti KC who helped during its revision.

\section{Conflict of interest}

SW and JEG work for Public Health England and FCS currently works for the National Audit Office. The views expressed do not represent the views of the above organisations.

\section{References}

1. Colonio-Salazar F B, Sipiyaruk K, White S, Gallagher J E. Key determinants of health and wellbeing of dentists within the UK: a rapid review of over two decades of research. Br Dent J 2019; 227: 127-136.

2. Hancocks S. Well, being a dentist. Br Dent J 2019; 227: 69 .

3. O'Selmo E, Collin V, Whitehead P. Dental associates' perceptions of their working environment: a qualitative study. Br Dent J 2019; 226: 955-962.

4. Collin V, Toon M, O'Selmo E, Reynolds L, Whitehead P. A survey of stress, burnout and well-being in UK dentists. BrDent J 2019; 226: 40-49.

5. Toon M, Collin V, Whitehead P, Reynolds L. An analysis of stress and burnout in UK general dental practitioners: subdimensions and causes. Br Dent J 2019; 226: 125-130.

6. NHS Digital. Dental Working Hours: 2014/15 and 2015/16 Motivation Analysis, Experimental Statistics. London: NHS Digital, 2016.

7. NHS Digital. Dental Working Hours: 2016/17 and 2017/18 Working Patterns, Motivation and Morale [PAS]. London: NHS Digital, 2018.

8. NHS Digital. Dentists' Working Patterns, Motivation and Morale-2018/19 and 2019/20. London: NHS Digital, 2020.

9. British Dental Association. The Psychosocial Working Conditions and Work-related stress among Community Dentists in the UK: Results from the 2014 Dentists' Wellbeing and Working Conditions surveys. London: BDA, 2015

10. Larbie J, Kemp M, Whitehead P. The Mental Health and Well-being of UK Dentists: being of UK Dentists: A Qualitative Study. London: BDA, 2017.

11. NHS Digital. Dental Working Hours - Working Patterns, Motivation and Morale 2016/2017 and 2017/2018. London: NHS Digital, 2018.

12. British Dental Association. Half of NHS young dentists heading for the exit. 2017. Available online at https:// bda.org/news-centre/press-releases/half-of-nhsyoung dentists-heading-for-the-exit (accessed October 2020).

13. British Dental Association. $75 \%$ of NHS dental practices now struggling to fill vacancies. 2019. Available online at https://bda.org/news-centre/press-releases/ Pages/75-per-cent-of-NHS-dental-practices-nowstruggling-to-fill-vacancies.aspx (accessed October 2020)

14. British Dental Association. Is Government serious about the survival of NHS dentistry? 2019. Available at https://bda.org/news-centre/blog/is-governmentserious-about-the-survival-of-nhs-dentistry (accessed May 2021).

15. Surman G, Goldacre M J, Lambert T W. UK-trained junior doctors' intentions to work in UK medicine: questionnaire surveys, three years after graduation. IR Soc Med 2017: 110: 493-500.

16. Moberly T. Health and wellbeing is the most common reason for doctors to take a break from training. $B M J$ 2018; DOI: 10.1136/bmj.k3097.

17. British Dental Association. Student Manifesto 20182020. 2018. Available at https://bda.org/news-centre/ 
blog/Documents/BDA-Student-Manifesto-2018.pdf (accessed May 2021).

18. Watt R G, Steele J G, Treasure E T, White D A, Pitts N B, Murray J J. Adult Dental Health Survey 2009 : implications of findings for clinical practice and oral health policy. Br Dent J 2013; 214: 71-75.

19. White D A, Tsakos G, Pitts N B et al. Adult Dental Health Survey 2009: common oral health conditions and their impact on the population. Br Dent J 2012; 213: 567-572.

20. The Health and Social Care Information Centre. Adult Dental Health Survey 2009 - Summary report and thematic series [NS]. London: HSCIC, 2011.

21. Steele J. Review of NHS Dental Services in England: an independent review. London: Department of Health, 2009.

22. General Dental Council. Registration report - March 2019. London: GDC, 2019.

23. Health Education England. Advancing Dental Care (ADC) Education \& Training Review. 2018. Available online at https://www.hee.nhs.uk/our-work/ advancing-dental-care (accessed October 2020).

24. Palinkas L A, Horwitz S M, Green C A, Wisdom J P, Duan N, Hoagwood K. Purposeful Sampling for Qualitative Data Collection and Analysis in Mixed Method Implementation Research. Adm Policy Ment Health 2015; 42: 533-544.

25. Ritchie J, Lewis J, Nicholls C M, Ormston R. Qualitative research practice: A guide for social science students and researchers. London: Sage, 2013.

26. NHS Digital. NHS Dental Statistics England 2015/16 London: NHS Digital, 2016.

27. Kemp M, Edwards H. The Financial Circumstances of Final Year Dental Undergraduates in the UK. London: BDA, 2014

28. D'Amour D, Oandasan I. Interprofessionality as the field of interprofessional practice and interprofessional education: An emerging concept. J Interprof Care 2005; 19 Suppl 1: 8-20.

29. Tong A, Sainsbury P, Craig J. Consolidated criteria for reporting qualitative research (COREQ): a 32-item checklist for interviews and focus groups. Int J Qual Health C 2007; 19: 349-357.

30. Flowers $\mathrm{P}$, Larkin $\mathrm{M}$, Smith J. Interpretative phenomenological analysis: Theory, method and research. London: Sage Publications Ltd, 2009.

31. Gilmour J, Stewardson D A. Morale of vocational dental practitioners in the United Kingdom. Br Dent J 2008; 204: E18.

32. Bretherton R, Chapman H R, Chipchase S. A study to explore specific stressors and coping strategies in primary dental care practice. Br Dent J 2016; 220: 471-478.

33. Humphris $G$ M Cooper $C L$ New stressors for GDPs in the past ten years: a qualitative study. Br Dent J 1998; 185: 404-406.
34. O'Selmo E, Collin V, Whitehead P. Dental associates' perceptions of their working environment: a qualitative study. Br Dent J 2019; 226: 955-962.

35. Myers H, Myers L. 'It's difficult being a dentist': Stress and health in the general dental practitioner. Br Dent $J$ 2004; 197: 89-93; discussion 83; quiz 100.

36. Chapman H R, Chipchase SY, Bretherton R. Understanding emotionally relevant situations in primary dental practice. 3. Emerging narratives. Br Dent 12015: 219: 491-496.

37. Hill K B, Burke F J T, Brown J et al. Dental practitioners and ill health retirement: a qualitative investigation into the causes and effects. Br Dent J 2010; 209: E8.

38. Newton J T, Gibbons D E. Levels of career satisfaction among dental healthcare professionals: comparison of dental therapists, dental hygienists and dental practitioners. Community Dent Health 2001; 18: 172-176.

39. Gorter R C, Freeman R. Burnout and engagement in relation with job demands and resources among dental staff in Northern Ireland. Community Dent Oral Epidemiol 2011; 39: 87-95.

40. Gilmour J, Stewardson D A, Shugars D A, Burke F J T. An assessment of career satisfaction among a group of general dental practitioners in Staffordshire. Br Dent J 2005; 198: 701-704.

41. Kay E J, Lowe J C. A survey of stress levels, selfperceived health and health-related behaviours of UK dental practitioners in 2005. Br Dent J 2008; 204: E19.

42. Jones L M, Cotter R, Birch K M. A review of occupationally-linked suicide for dentists. N Z Dent 2016; 112: 39-46.

43. Rogers C, Malone K M. Stress in Irish dentists: developing effective coping strategies. J Ir Dent Assoc 2009: 55: 304-307.

44. Gorter R C, Albrecht G, Hoogstraten J, Eijkman M A. Professional burnout among Dutch dentists. Community Dent Oral Epidemiol 1999: 27: 109-116.

45. Ab-Murat N, Mason L, Kadir R A, Yusoff N. Selfperceived mental well-being among Malaysian dentists. Int J Occup Saf Ergon 2018; 24: 233-239.

46. Meyerson J, Gelkopf M, Eli I, Uziel N. Burnout and professional quality of life among Israeli dentists: the role of sensory processing sensitivity. Int Dent J 2020; 70: 29-37.

47. Clough B A, Ireland M J, Leane S, March S. Stressors and protective factors among regional and metropolitan Australian medical doctors: A mixed methods investigation. J Clin Psychol 2020; 76: 1362-1389.

48. Robertson H D, Elliott A M, Burton C et al. Resilience of primary healthcare professionals: a systematic review. BrJ Gen Pract 2016; DOI: 10.3399/bjgp16X685261.

49. Massachusetts Medical Society, Massachusetts Health and Hospital Association, Harvard T. H. Chan School of Public Health and Harvard Global Health Institute. A Crisis in Health Care: a call to action on Physician Burnout. Boston: Harvard Global Health Institute, 2019.
50. Rimmer A. Covid-19: Doctors may quit without proper post-pandemic support, defence body warns. BMJ 2020; DOI: 10.1136/bmj.m2476.

51. National Academy of Medicine. Action Collaborative on Clinician Well-Being and Resilience. 2017. Available online at https://nam.edu/initiatives/clinician-resilienceand-well-being/ (accessed May 2021)

52. Brigham T, Barden C, Dopp A L et al. Factors Affecting Clinician Well-Being and Resilience - Conceptual Model. Washinton DC: National Academy of Medicine, 2018.

53. Oldfield E. Plymouth's NHS dentists crisis - experts demand action. Plymouth Herald (Plymouth) 2020 January 14.

54. Gallagher JE. The Future Oral and Dental Workforce for England: Liberating human resources to serve the population across the life-course. 2019. Available at https://www.hee.nhs.uk/sites/default/files/documents/ FDWF\%20Report\%20-\%207th\%20March\%202019.pdf (accessed May 2021).

55. World Health Organisation. Coronavirus disease (COVID19) pandemic. 2020. Available online at https://www. who.int/emergencies/diseases/novel-coronavirus-2019 (accessed May 2021)

56. Armstrong M. Coronavirus: Bringing dentistry back from the brink. 2020. Available at https://bda.org/newscentre/blog/Pages/Coronavirus-Bringing-dentistry-backfrom-the-brink.aspx (accessed September 2020).

57. Wilson R F, Coward PY, Capewell J, Laidler T L, Rigby A C, Shaw T J. Perceived sources of occupational stress in general dental practitioners. Br Dent J 1998; 184: 499-502.

58. British Dental Association. Salaried Primary Dental Care Service Morale Survey. London: BDA, 2011.

59. Edwards $\mathrm{H}, \mathrm{Kemp} M$. Morale and Motivation of Dentists in the Salaried Primary Dental Care Service. London: BDA, 2012.

60. Boyles J D, Ahmed B. Does student debt affect dental students' and dentists' stress levels? Br Dent J 2017; 223: 601.

61. McGregor S. Neoliberalism and health care. Int J Consum Stud 2001; 25: 82-89.

62. NHS England. NHS England Long Term Plan. London: NHS England, 2019.

63. Francis T, Hoefel F. 'True Gen': Generation Z and its implications for companies. 2018. Available at https:// www.mckinsey.com/industries/consumer-packagedgoods/our-insights/true-gen-generation-z-and-itsimplications-for-companies\# (accessed May 2021).

64. Armstrong M. The dental profession's mental health crisis: what needs to happen. 2020. Available at https://bda.org/ news-centre/blog/Pages/The-dental-professions-mentalhealth-crisis.aspx (accessed September 2020).

65. World Health Organisation. Global strategy on human resources for health: Workforce 2030. 2016. Available online at http://www.who.int/hrh/resources/pub_ globstrathrh-2030/en/ (accessed May 2021). 